\title{
Emerging and Established Model Systems for Endosperm Weakening
}

\author{
G. Leubner-Metzger, B. Kucera and K. Müller \\ Institute for Biology II, Botany/Plant Physiology, Albert-Ludwigs-University \\ Freiburg, Schänzlestr. 1, D-79104 Freiburg i. Br., Germany
}

\section{Abstract}

Endosperm rupture is the main germination-limiting process in members of the Asteraceae (e.g. lettuce (Lactuca sativa L.)) and Solanaceae (e.g. tomato (Lycopersicon esculentum Mill.) and tobacco (Nicotiana tabacum L.). About four decades ago a 'hatching enzyme' was proposed to cause endosperm weakening (i.e. a decline in the mechanical resistance of the micropylar endosperm), which is likely to be essential for seeds to complete germination. Although, there are established model systems among Asteraceae and Solanaceae for endosperm weakening, its molecular mechanism(s) still remain(s) a mystery. No single 'hatching enzyme' or universal molecular mechanism has been demonstrated explicitly. For the time being, the provisional conclusion is that endosperm weakening is likely to be achieved by the collaborative or successive action of several distinct molecular mechanisms. The knowledge gained from these established model systems will be compared and discussed. However, consideration of their severe experimental limitations shows that there is an urgent need for novel model systems. Such an optimal system has been recently found within the Brassicaceae. In this emerging model system for endosperm weakening, a complete study of the process is possible on each experimental level, from the direct measurement of the weakening by 'puncture force' to molecular investigations (e.g. proteome and genome transcriptome analyses).

\section{Introduction}

A major reason for the evolutionary success of the angiosperms is the 'invention' of seeds with double fertilization (Friedman, 1998; Judd et al., 2002). In a typical angiosperm seed the diploid embryo is surrounded by two covering layers: (i) the triploid endosperm (i.e. nutritive tissue, living cells); and (ii) the diploid testa (i.e. the seed coat, maternal tissue, dead cells). Depending on the species, the endosperm is either maintained or obliterated during seed development. The evolutionary trend is towards cotyledon storage and endospermless seeds at maturity. Endosperm 
development and its function as nutritive tissue have been studied thoroughly (Jacobsen et al., 1995; Friedman, 1998; Baskin and Baskin, 2004). In contrast, little is known about the function of the endosperm as a constraint during endospermlimited germination and coat-imposed dormancy (Bewley, 1997; Leubner-Metzger, 2003). Seed germination is a complex physiological process, water uptake by imbibition is followed by embryo growth, and radicle protrusion through all seedcovering layers is considered as the completion of germination. Environmental factors and plant hormones (e.g. gibberellins (GA), abscisic acid (ABA), brassinosteroids and ethylene) are regulators of germination and/or dormancy (Koornneef et al., 2002; Kucera et al., 2005).

The mature seeds of most angiosperms have a more or less abundant endosperm layer (Fig. 20.1). Mature angiosperm seeds differ in their 'embryo to seed' (E/S) ratios (Martin, 1946; Forbis et al., 2002). Low E/S values, due to abundant endosperm tissue and tiny embryos, are typical for mature seeds of basal angiosperms (Fig. 20.1). High E/S values, due to obliterated endosperm tissue and the predominance of cotyledon storage, are evident in mature seeds of higher angiosperms of the rosid clade. Typical examples are the more or less endospermless Brassicaceae seeds with E/S values $\sim 0.9$ (Fig. 20.1). Although the mature seeds of Brassica species are completely endospermless, a single cell layer of endosperm is present in mature seeds of Arabidopsis (Arabidopsis thaliana (L.) Heynh.) (Liu et al., 2005). E/S values between 0.4 and 0.5 are typical for mature seeds of higher angiosperms of the asterid clade (e.g. the Solanaceae), with abundant endosperm and additional embryo storage (Fig. 20.1; Bewley, 1997; Hilhorst et al., 1998; Koornneef et al., 2002; Leubner-Metzger, 2003).

The distribution of the different seed types and the $\mathrm{E} / \mathrm{S}$ values in a modern phylogenetic tree (Fig. 20.1) support the following evolutionary seed trends (Martin, 1946; Forbis et al., 2002; Baskin and Baskin, 2004): (i) in mature seeds of primitive angiosperms a small embryo is embedded in abundant endosperm tissue, and such seed types are prevalent among basal angiosperms; (ii) the general evolutionary trend within the higher angiosperms (i.e. core eudicots) is by the Solanaceae-like endospermic seed types of many asterids towards Brassicaceae-like more or less endospermless seed types of many rosids with storage cotyledons; and (iii) in addition to these general seed trends there are clade-specific differences. It has been proposed that endospermless seeds were 'invented' several times independently during evolution (Baskin and Baskin, 2004). Thus, our knowledge on endosperm abundance in mature seeds is quite complete.

In contrast, we do not know how endosperm-limited germination and/or endosperm-enhanced dormancy evolved during angiosperm seed phylogeny. Figure 20.1 shows (in bold) the few clades with at least some experimental evidence for seeds where the endosperm is important as a germination barrier (almost nothing is known for many rosid species). An exception is the work on perisperm weakening of Cucurbitaceae seeds (e.g. Welbaum et al., 1995; Yim and Bradford, 1998). Established model systems for endosperm-limited germination (Fig. 20.1) are exclusively asterid species (Bewley, 1997; Hilhorst et al., 1998; Leubner-Metzger, 2003; da Silva et al., 2004, 2005): lettuce (Lactuca sativa L., Asteraceae, Asterales), tomato (Lycopersicon esculentum Mill., Solanaceae, Solanales), tobacco (Nicotiana tabacum L., Solanaceae, Solanales) and coffee (Coffea arabica L., Rubiaceae, Gentianales). Endosperm weakening, a decline 


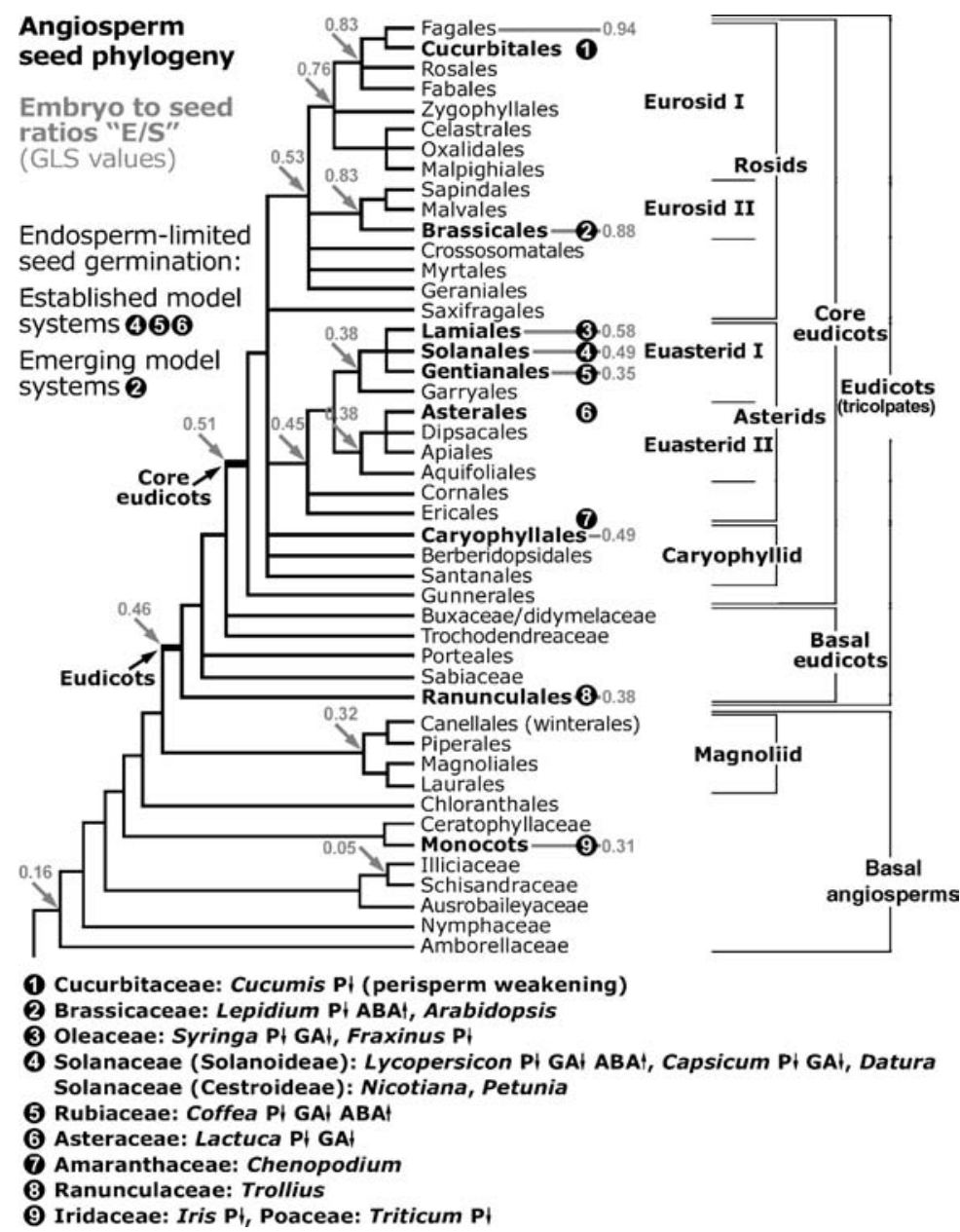

Fig. 20.1. Angiosperm seed phylogeny. Grey numbers in the phylogenetic tree represent 'embryo to seed' (E/S) ratios expressed as generalized least squares (GSL) values (Forbis et al., 2002). Clades with experimental evidence for endosperm weakening are in bold and numbered; some of these provide model systems. $\mathrm{\downarrow} \downarrow$ : endosperm weakening during germination was measured as decreasing puncture force; GA $\downarrow$ : endosperm weakening promoted by GA; and ABA $\uparrow$ : endosperm weakening inhibited by $A B A$.

in the mechanical resistance of the micropylar endosperm (i.e. endosperm covering the radicle tip), appears to be a prerequisite for the germination of these species. Endosperm weakening can be measured directly by the puncture-force method (see Müller et al., Chapter 30, this volume). Puncture-force experiments are not possible with seeds as small as tobacco or Arabidopsis (Fig. 20.2), but have been performed for larger asterid seeds of the Asterales (Pavlista and Haber, 1970; Tao and Khan, 1979), Gentianales (da Silva et al., 2004, 2005), Solanales (Watkins and Cantliffe, 1983; 


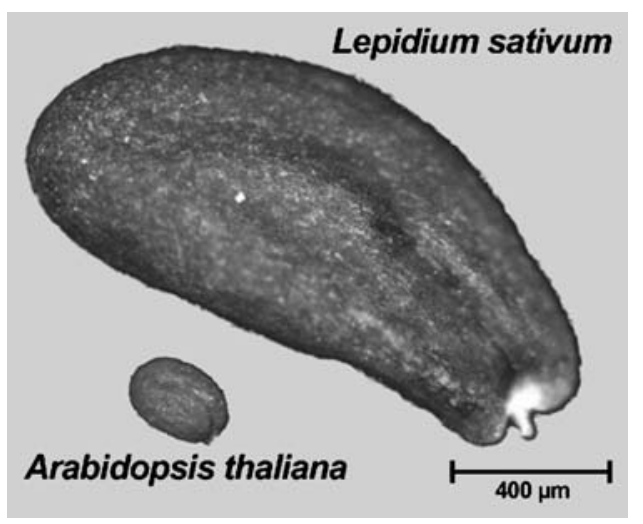

Fig. 20.2. Size comparison of dry seeds of Lepidium sativum and Arabidopsis thaliana, two closely related species of the Brassicoideae subfamily of the Brassicaceae.

Toorop et al., 2000; Wu et al., 2000) and Laminales (Junttila, 1973; Finch-Savage and Clay, 1997). All these experiments showed a decline in the puncture force of

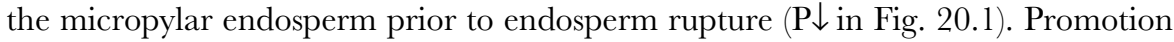
of endosperm weakening by GA appears to be a general phenomenon (GA $\downarrow$ in Fig. 20.1). Thus, endosperm weakening prior to endosperm rupture seems to be widespread among asterid seeds, but has not been investigated in rosid seeds.

\section{ABA Inhibition of Seed Germination: ABA Inhibits Endosperm Rupture, but not Testa Rupture}

Another phenomenon of endospermic seeds is that endosperm rupture is inhibited by ABA (Karssen, 1976; Finch-Savage and McQuistan, 1991; Toorop et al., 2000; Petruzzelli et al., 2003). In the established model systems of the asterid clade it has been shown that ABA inhibits endosperm rupture, at least in part, by acting in an inhibitory manner on the micropylar endosperm ( $\mathrm{Ni}$ and Bradford, 1993; LeubnerMetzger, 2003; da Silva et al., 2004). The germination of intact tomato seeds is inhibited by $10-100 \mu \mathrm{M}$ ABA, but surgical removal of the micropylar cap permits germination (i.e. initial embryo elongation) even in the presence of $1000 \mu \mathrm{M}$ ABA (Liptay and Schopfer, 1983). The Solanaceae family can be divided into two large subgroups (Judd et al., 2002; Petruzzelli et al., 2003): (i) the Solanoideae (e.g. Capsicum, Lycopersicon and Datura); and (ii) the Cestroideae (e.g. Nicotiana and Petunia). In Solanoideae-type seeds, the micropylar covering layers, testa and endosperm form a caplike structure (i.e. a micropylar cap) (Hilhorst et al., 1998; Toorop et al., 2000). A visible distinction between testa rupture and endosperm rupture is not possible for Solanoideae-type seeds. A typical feature of Cestroideae-type seeds like tobacco is a two-step germination with a visible distinction between testa rupture and endosperm rupture (Fig. 20.3; Leubner-Metzger, 2003; Petruzzelli et al., 2003).

Two-step germination with separate testa and endosperm rupture is widespread over the entire phylogenetic tree and has been described for many species, including Trollius (Ranunculaceae, Ranunculales, basal eudicots) (Hepher and Roberts, 1985), Chenopodium (Amaranthaceae, Caryophyllales, caryophyllids) (Karssen, 1976) and 

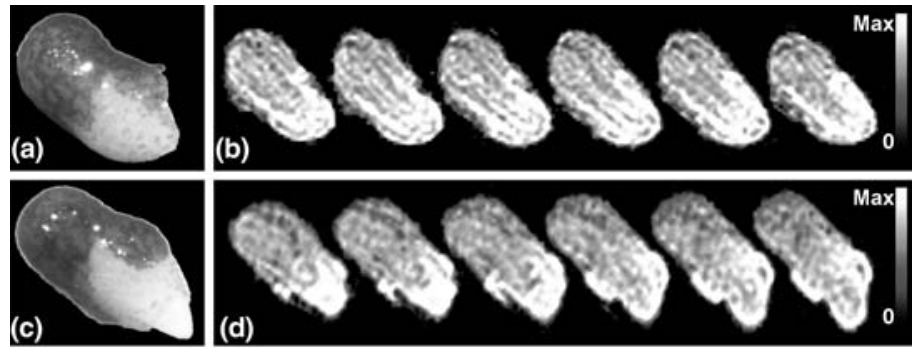

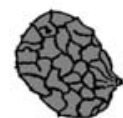

(e)

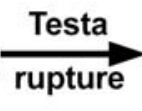

rupture

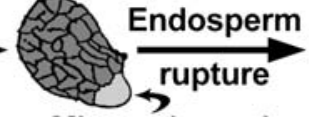

Micropylar endosperm $\checkmark$ tRadicle tip

Fig. 20.3. Two-step germination and water uptake of tobacco seeds. (a, b and e) Testa rupture is followed by (c, $d$ and e) endosperm rupture and subsequent seedling growth. (b and d) Nuclear magnetic resonance (NMR) images show that the micropylar endosperm and the radicle are major sites of water uptake. White: maximum water abundance; black: minimum water abundance (see NMR images in Manz et al., 2005 for details).

Nicotiana (Cestroideae, Solanaceae, Solanales, asterids) (Leubner-Metzger, 2003). Recently, it has also been found in the Brassicaceae species, Arabidopsis thaliana L. (Heynh.) (Liu et al., 2005) and lepidium (Lepidium sativum L.) (see Müller et al., Chapter 30, this volume). Separate testa and endosperm rupture is therefore a feature of endospermic seeds within the rosid clade. It was also found that ABA inhibits endosperm rupture, but not testa rupture, of Arabidopsis and lepidium (see Müller et al., Chapter 30 , this volume). In agreement with this, ABA also does not inhibit testa rupture of endospermless Brassica seeds (Schopfer and Plachy, 1984). Separate testa rupture and endosperm rupture are an important experimental advantage of tobacco seeds when compared with tomato seeds. It has helped considerably to assign different enzymes, transcription factors and plant hormones to their target sites (LeubnerMetzger, 2003).

Although ABA inhibits the embryo growth potential and the transition to postgermination growth, it does not inhibit initial water uptake by imbibition or initial embryo elongation (Liptay and Schopfer, 1983; Schopfer and Plachy, 1984; Homrichhausen et al., 2003; Manz et al., 2005). We measured the regulation of water uptake of germinating tobacco seeds spatially and temporally by in vivo ${ }^{1} \mathrm{H}$ nuclear magnetic resonance $\left({ }^{1} \mathrm{H}-\mathrm{NMR}\right)$ microimaging and ${ }^{1} \mathrm{H}-\mathrm{MAS}$ NMR spectroscopy (Manz et al., 2005). These non-destructive state-of-the-art methods show that water distribution in the water uptake phases II and III is inhomogeneous (Fig. 20.3). The micropylar seed end is the major entry point of water. The micropylar endosperm and the radicle show the highest hydration. ABA specifically inhibits endosperm rupture and phase III water uptake, but does not alter the spatial and temporal pattern of phase II water uptake. Taken together, these findings demonstrate that the micropylar endosperm is a main target for the ABA inhibition of endosperm rupture. 


\section{ABA, Endosperm Hydrolases and Endosperm Weakening in Established Asterid Model Systems}

Direct measurements of the effect of ABA on endosperm weakening by punctureforce experiments have been published only for the asterid species like coffee and tomato (Toorop et al., 2000; Wu et al., 2000; da Silva et al., 2004, 2005). The coffee embryo is enveloped by an endosperm tissue and surrounded by an endocarp (da Silva et al., 2004). The endosperm is composed of a hard greenish tissue with polyhedral cells, is isodiametrically divided into a hard external endosperm and a soft internal endosperm, and belongs to the nuclear type. Endosperm weakening was measured in imbibed seeds with the endocarp mechanically removed. Endosperm weakening in coffee is biphasic. The first phase of endosperm weakening is ABAinsensitive, which is followed by the second phase that is inhibited by ABA. This second phase accounts for $\sim 53 \%(420 \mathrm{mN})$ of the total difference in puncture force (da Silva et al., 2004). Endosperm weakening of tomato is also biphasic, with a first phase that is ABA-insensitive. In this case the second phase, which is inhibited by $\mathrm{ABA}$, has been reported to account for $\sim 6 \%(30 \mathrm{mN}$; Wu et al., 2000) or $\sim 24 \%$ (80 mN; Toorop et al., 2000) of the total tomato micropylar cap weakening. These results were obtained by incubating whole seeds in a medium with and without $\mathrm{ABA}$, and dissecting the seeds when puncture-force measurements were performed (Groot and Karssen, 1987, 1992; Toorop et al., 2000; Wu et al., 2000). If micropylar caps are dissected from tomato seeds prior to the onset of endosperm weakening $(3 \mathrm{~h})$, a further $24 \mathrm{~h}$ incubation of isolated micropylar caps in medium with GA results in endosperm weakening (i.e. puncture force decreases by $170 \mathrm{mN}$ ), whereas incubation of isolated micropylar caps in a GA-plus ABA-containing medium inhibits endosperm weakening completely (Groot and Karssen, 1992). Although ABA clearly inhibits the second-phase endosperm weakening of coffee, the situation in tomato is less clear.

The micropylar cap of tomato seeds consists of endosperm and testa. No visible distinction between testa rupture and endosperm rupture is possible in tomato. Although the micropylar testa of tomato seeds accounts for only $20 \%$ of the initial puncture force and does not weaken during the first phase of the biphasic micropylar cap weakening, a significant decline in testa puncture force occurs during the second (i.e. ABA-sensitive) phase of tomato micropylar cap weakening just prior to radicle protrusion (Groot and Karssen, 1987). Tomato micropylar cap weakening is also highly dependent on the physiological seed stage. Endosperm weakening and endosperm rupture were delayed in freshly harvested tomato seeds when compared with after-ripened seeds (Groot and Karssen, 1992). ABA deficiency of the sit ${ }^{\text {w }}$ tomato mutant replaced this after-ripening effect, and micropylar cap weakening and endosperm rupture of freshly harvested and after-ripened $s i t^{w}$ seeds were equal. The ABA-deficient sit ${ }^{\text {w }}$ tomato mutant has a thinner testa (i.e. one cell layer thick) compared with wild-type seeds (i.e. 3-4 cell layers thick) and is therefore a testa mutant (Hilhorst and Downie, 1995). A species with separate testa rupture and endosperm rupture would provide a seed model system with experimental advantages for studying endosperm weakening (see Müller et al., Chapter 30, this volume).

Little is known about the molecular mechanisms of endosperm weakening (Bewley, 1997; Toorop et al., 2000; Leubner-Metzger, 2003; da Silva et al., 2004, 
2005). Ikuma and Thimann (1963) proposed the 'hatching hypothesis' of seed biology as 'the final step in the germination control process is the production of an enzyme whose action enables the tip of the radicle to penetrate through the coat'. Experiments to identify this 'hatching enzyme' have been conducted in a variety of species and have provided evidence for the contribution of various cell wall-modifying proteins (e.g. endo- $\beta$-1,4-mannanases, endo- $\beta$-1,3-glucanases and expansins) (Bewley, 1997; Hilhorst et al., 1998; Koornneef et al., 2002; LeubnerMetzger, 2003). Expression of endo- $\beta$-1,4-mannanase in the micropylar endosperm of coffee is associated with the second phase of endosperm weakening and is inhibited by ABA (da Silva et al., 2004). In contrast, endo- $\beta-1,4-$ mannanase in tomato seeds is associated with the first phase of endosperm weakening and is not inhibited by ABA (Nonogaki et al., 2000; Toorop et al., 2000). Expression of endo- $\beta-1$, 3-glucanase in the micropylar endosperm, its inhibition by ABA and the inhibition of endosperm rupture by ABA is widespread among the Solanaceae $(\mathrm{Wu}$ et al., 2000; Leubner-Metzger, 2003; Petruzzelli et al., 2003). The ABA inhibition of endosperm rupture is partially reverted in transgenic tobacco seeds that overexpress endo- $\beta$-1,3-glucanase in the seed-covering layers under the control of an ABA-inducible transgene promoter (Leubner-Metzger and Meins, 2000; LeubnerMetzger, 2002; Manz et al., 2005). This directly proves that endo- $\beta$-1,3-glucanase is causally involved in promoting endosperm rupture, but it is not the sole 'hatching enzyme' (Leubner-Metzger, 2003). Conclusive evidence for a sole 'hatching enzyme' has not yet been found. In his review, Bewley (1997) stated that 'endosperm weakening is likely to be essential for some seeds to complete germination, how it is achieved remains a mystery'. Taken together, these findings support the view that germination control by the seed-covering layers is achieved by the collaborative or successive action of several cell wall-modifying proteins and various molecular mechanisms (Leubner-Metzger, 2003). The intriguing issue that arises is that there might be evolutionary conserved molecular mechanisms as well as species-specific adaptations for endosperm weakening.

\section{ABA, Endosperm Weakening and Novel Molecular Mechanisms Studied in Emerging Rosid Model Systems}

Within the rosid clade, there is prevalence for more or less endospermless seeds (Fig. 20.1). There are also several examples of endospermic rosid seeds (O'Brien and McCully, 1969), but not a single publication about rosid endosperm weakening. A new model system for endosperm-limited germination with molecular phylogenetic placement within the rosid clade is therefore a necessity (Mandoli and Olmstead, 2000; Soltis and Soltis, 2003). The genome of the rosid model plant Arabidopsis is completely sequenced. The placement of a novel rosid model system in close proximity with this 'molecular' model plant allows successful utilization of the Arabidopsis databases for modern molecular methods like transcriptomics and proteomics (Mandoli and Olmstead, 2000; Hall et al., 2002).

Lepidium is closely related to Arabidopsis; both belong to Brassicoideae subfamily of Brassicaceae. Both species have separate testa and endosperm rupture, and ABA inhibits endosperm rupture, but not testa rupture (Liu et al., 2005; see Müller et al., 
Chapter 30, this volume). Arabidopsis seeds are too small to perform puncture-force measurements (Fig. 20.2). These measurements show that endosperm weakening occurs prior to lepidium endosperm rupture (see Müller et al., Chapter 30, this volume). This endosperm weakening is inhibited by ABA. In future, we want to exploit these experimental advantages to investigate the molecular mechanisms that regulate lepidium endosperm weakening. Lepidium is an emerging model system for studying endosperm weakening. Reactive oxygen species are a novel molecular mechanism for endosperm weakening and embryo expansion during seed germination (see Müller et al., Chapter 30, this volume).

\section{Acknowledgements}

We gratefully acknowledge travel support by the Deutsche Forschungsgemeinschaft (to G. Leubner-Metzger), the seed company Ernst Benary Samenzucht GmbH, Hann. Münden, Germany (to B. Kucera) and the Wissenschaftliche Gesellschaft Freiburg (to K. Müller) for attending the Eighth International Seed Workshop in Brisbane, Australia, May 2005. Our research is supported by grants from the Deutsche Forschungsgemeinschaft and the Wissenschaftliche Gesellschaft Freiburg.

\section{References}

Baskin, J.M. and Baskin, C.C. (2004) A classification system for seed dormancy. Seed Science Research $14,1-16$.

Bewley, J.D. (1997) Breaking down the walls: a role for endo- $\beta$-mannanase in release from seed dormancy? Trends in Plant Science 2, 464-469.

da Silva, E.A.A., Toorop, P.E., van Aelst, A.C. and Hilhorst, H.W.M. (2004) Abscisic acid controls embryo growth potential and endosperm cap weakening during coffee (Coffea arabica cv. Rubi) seed germination. Planta 220, 251-261.

da Silva, E.A.A., Toorop, P.E., Nijsse, J., Bewley, J.D. and Hilhorst, H.W.M. (2005) Exogenous gibberellins inhibit coffee (Coffea arabica $\mathrm{cv}$. Rubi) seed germination and cause cell death in the embryo. Fournal of Experimental Botany 413, 1029-1038.

Finch-Savage, W.E. and Clay, H.A. (1997) The influence of embryo restraint during dormancy loss and germination of Fraxinus excelsior seeds. In: Ellis, R.H., Black, M., Murdoch, A.J. and Hong, T.D. (eds) Basic and Applied Aspects of Seed Biology. Kluwer Academic Publishers, Dordrecht, The Netherlands, pp. 245-253.

Finch-Savage, W.E. and McQuistan, C.I. (1991) Abscisic acid: an agent to advance and synchronise germination for tomato (Lycopersicon esculentum Mill.) seeds. Seed Science and Technology 19, 537-544.

Forbis, T.A., Floyd, S.K. and deQueiroz, A. (2002) The evolution of embryo size in angiosperms and other seed plants: implications for the evolution of seed dormancy. Evolution 56, 2112-2125.

Friedman, W.E. (1998) The evolution of double fertilization and endosperm: an "historical" perspective. Sexual Plant Reproduction 11, 6-16.

Groot, S.P.C. and Karssen, C.M. (1987) Gibberellins regulate seed germination in tomato by endosperm weakening: a study with gibberellin-deficient mutants. Planta 171, 525-531.

Groot, S.P.C. and Karssen, C.M. (1992) Dormancy and germination of abscisic acid-deficient tomato seeds. Plant Physiology 99, 952-958.

Hall, A.E., Fiebig, A. and Preuss, D. (2002) Beyond the Arabidopsis genome: opportunities for comparative genomics. Plant Physiology 129, 1439-1447. 
Hepher, A. and Roberts, J.A. (1985) The control of seed germination in Trollius ledebouri: the breaking of dormancy. Planta 166, 314-320.

Hilhorst, H.W.M. and Downie, B. (1995) Primary dormancy in tomato (Lycopersicon esculentum cv. Moneymaker): studies with the sitiens mutant. Fournal of Experimental Botany 47, 89-97.

Hilhorst, H.W.M., Groot, S.P.C. and Bino, R.J. (1998) The tomato seed as a model system to study seed development and germination. Acta Botanica Neerlandica 47, 169-183.

Homrichhausen, T.M., Hewitt, J.R. and Nonogaki, H. (2003) Endo- $\beta$-mannanase activity is associated with the completion of embryogenesis in imbibed carrot (Daucus carota L.) seeds. Seed Science Research 13, 219-227.

Ikuma, H. and Thimann, K.V. (1963) The role of the seed-coats in germination of photosensitive lettuce seeds. Plant and Cell Physiology 4, 169-185.

Jacobsen, J.V., Gubler, F. and Chandler, P.M. (1995) Gibberellin action in germinated cereal grains. In: Davies, P.J. (ed.) Plant Hormones. Kluwer Academic Publishers, Dordrecht, The Netherlands, pp. 246-271.

Judd, W.S., Campbell, C.S., Kellog, E.A., Stevens, P.F. and Donoghue, M.J. (2002) Plant Systematics: A Phylogenetic Approach, 2nd edn. Sinauer Associates, Sunderland, Massachusetts.

Junttila, O. (1973) The mechanism of low temperature dormancy in mature seeds of Syringa species. Plant Physiology 29, 256-263.

Karssen, G.M. (1976) Uptake and effect of abscisic acid during induction and progress of radicle growth in seeds of Chenopodium album. Physiologia Plantarum 36, 259-263.

Koornneef, M., Bentsink, L. and Hilhorst, H. (2002) Seed dormancy and germination. Current Opinion in Plant Biology 5, 33-36.

Kucera, B., Cohn, M.A. and Leubner-Metzger, G. (2005) Plant hormone interactions during seed dormancy release and germination. Seed Science Research 15, 281-307.

Leubner-Metzger, G. (2002) Seed after-ripening and over-expression of class I $\beta$-1,3-glucanase confer maternal effects on tobacco testa rupture and dormancy release. Planta 215, 959-968.

Leubner-Metzger, G. (2003) Functions and regulation of $\beta$-1,3-glucanase during seed germination, dormancy release and after-ripening. Seed Science Research 13, 17-34.

Leubner-Metzger, G. and Meins, F. Jr (2000) Sense transformation reveals a novel role for class I $\beta$-1,3-glucanase in tobacco seed germination. The Plant fournal 23, 215-221.

Liptay, A. and Schopfer, P. (1983) Effect of water stress, seed coat restraint, and abscisic acid upon different germination capabilities of two tomato lines at low temperature. Plant Physiology 73, 935-938.

Liu, P.-P., Koizuka, N., Homrichhausen, T.M., Hewitt, J.R., Martin, R.C. and Nonogaki, H. (2005) Large-scale screening of Arabidopsis enhancer-trap lines for seed germination-associated genes. The Plant fournal 41, 936-944.

Mandoli, D.F. and Olmstead, R. (2000) The importance of emerging model systems in plant biology. Fournal of Plant Growth Regulation 19, 249-252.

Manz, B., Müller, K., Kucera, B., Volke, F. and Leubner-Metzger, G. (2005) Water uptake and distribution in germinating tobacco seeds investigated in vivo by nuclear magnetic resonance imaging. Plant Physiology 138, 1538-1551.

Martin, A.C. (1946) The comparative internal morphology of seeds. The American Midland Naturalist 36, 513-660.

Ni, B.R. and Bradford, K.J. (1993) Germination and dormancy of abscisic acid-deficient and gibberellindeficient mutant tomato (Lycopersicon esculentum) seeds: sensitivity of germination to abscisic acid, gibberellin, and water potential. Plant Physiology 101, 607-617.

Nonogaki, H., Gee, O.H. and Bradford, K.J. (2000) A germination-specific endo- $\beta$-mannanase gene is expressed in the micropylar endosperm cap of tomato seeds. Plant Physiology 123, 1235-1245.

O'Brien, T.P. and McCully, E. (1969) Plant Structure and Development: A Pictorial and Physiological Approach. Macmillan, Toronto, Ontario, Canada.

Pavlista, A.D. and Haber, A.H. (1970) Embryo expansion without protrusion in lettuce seeds. Plant Physiology 45, 636-637. 
Petruzzelli, L., Müller, K., Hermann, K. and Leubner-Metzger, G. (2003) Distinct expression patterns of $\beta$-1,3-glucanases and chitinases during the germination of Solanaceous seeds. Seed Science Research 13, 139-153.

Schopfer, P. and Plachy, C. (1984) Control of seed germination by abscisic acid. II. Effect on embryo water uptake in Brassica napus L. Plant Physiology 76, 155-160.

Soltis, D.E. and Soltis, P.S. (2003) The role of phylogenetics in comparative genetics. Plant Physiology 132, 1790-1800.

Tao, K.-L. and Khan, A.A. (1979) Changes in the strength of lettuce endosperm during germination. Plant Physiology 63, 126-128.

Toorop, P.E., van Aelst, A.C. and Hilhorst, H.W.M. (2000) The second step of the biphasic endosperm cap weakening that mediates tomato (Lycopersicon esculentum) seed germination is under control of ABA. Fournal of Experimental Botany 51, 1371-1379.

Watkins, J.T. and Cantliffe, D.J. (1983) Mechanical resistance of the seed coat and endosperm during germination of Capsicum annuum at low temperatures. Plant Physiology 72, 146-150.

Welbaum, G.E., Muthui, W.J., Wilson, J.H., Grayson, R.L. and Fell, R.D. (1995) Weakening of muskmelon perisperm envelope tissue during germination. Fournal of Experimental Botany 46, 391-400.

Wu, G.-T., Leubner-Metzger, G., Meins, F. Jr and Bradford, K.J. (2000) Class I $\beta$-1,3-glucanase and chitinase are expressed in the micropylar endosperm of tomato seeds prior to radicle emergence. Plant Physiology 126, 1299-1313.

Yim, K.O. and Bradford, K.J. (1998) Callose deposition is responsible for apoplastic semipermeability of the endosperm envelope of muskmelon seeds. Plant Physiology 118, 83-90. 